\title{
HYBRID ADAPTIVE CONTROL OF CSTR USING POLYNOMIAL SYNTHESIS AND POLE-PLACEMENT METHOD
}

\author{
Jiri Vojtesek, Petr Dostal and Roman Prokop \\ Faculty of Applied Informatics \\ Tomas Bata University in Zlin \\ Nad Stranemi 4511, 76005 Zlin, Czech Republic \\ E-mail: \{vojtesek,dostalp\}@fai.utb.cz
}

\section{KEYWORDS}

Adaptive control, Polynomial approach, Pole-placement method, Recursive identification, CSTR

\begin{abstract}
This paper deals with simulation studies of the adaptive control on the continuous stirred tank reactor (CSTR) as a typical chemical equipment with nonlinear behaviour and continuously distributed parameters. Mathematical model of this reactor is described by the set of two nonlinear ordinary differential equations (ODE). The simulation of the steady-state and dynamics results in optimal working point and external linear model (ELM) which is used in adaptive control. Adaptive approach here is based on the recursive identification of ELM and parameters of the controller are recomputed in each step too. The polynomial approach together with the poleplacement method and spectral factorization satisfies basic control requirements although the system has negative control properties.
\end{abstract}

\section{INTRODUCTION}

The most of the proceses in technical praxis has nonlinear behaviour and conventional control methods could lead to unoptimal or unwanted output responses. However, adaptive control (Åström 1989) is one way how to deal with the negative control properties such as non-minimum phase behaviour, nonlinearity, time delays etc.

There are several adaptive approaches. The one used in this work is based on the choice of an External Linear Model (ELM) parameters of which are recomputed recursively during the control (Bobal et al. 2005). It means that the steady-state and dynamic analyses need to be done for contructing of optimal ELM.

The ELM could be chosen from the range of the continuous-time or discrete-time models. Disadvantage of the continuous-time ELM is difficult on-line recursive identification. On the other hand, external delta models (Middleton and Goodwin 2004) used here for parameter estimation belong to the range of discrete models but parameters of these models approach up to some assumptions to their continuous-time counterparts (Stericker and Sinha 1993). Well known and widely used Ordinary recursive least squares method (Fikar and Mikles 1999) was used for parameter estimation during the control.
The polynomial method (Kucera 1993) used for controller synthesis together with the pole-placement method ensures basic control requirements such as stability, reference signal tracking and disturbance attenuation. Two control system configurations (Grimble 1994) were used - the first with one degreeof-freedom (1DOF) which has regulator only in feedback part and the second with two degrees-offreedom (2DOF) with feedback and feedforward parts. The resulting controllers are hybrid because polynomial synthesis is made for continuous-time but recursive identification runs on the delta-model, which belongs to the class of discrete-time models.

The Continuous Stired Tank Reactor (CSTR) which is an equipment widely used in chemical industry for its good control properties. Mathematical model of this plant is described by the set of two nonlinear ordinary differential equations which are solved numerically (Ingham et al. 2000). The system has two suitable input variables - volumetric flow rates of reactant and cooling and two outputs - product's temperature and concentration.

The article provides several control simulation studies on the CSTR, all of them were done on mathematical simulation software Matlab, version 6.5.1.

\section{MODEL OF THE PLANT}

The controlled process here is represented by the continuous stirred tank reactor (CSTR) and its scheme can be found in Figure 1.

Due to the simplification we expect that reactant is perfectly mixed and reacts to the final product with the concentration $c_{A}(t)$. The heat produced by the reaction is represented by the temperature of the reactant $T(t)$. Furthermore we also expect that volume, heat capacities and densities are constant during the control.

A mathematical model of this system is derived from the material and heat balances of the reactant and cooling. The resulted model is then a set of two Ordinary Differential Equations (ODEs) (Gao et al. 2002):

$$
\begin{gathered}
\frac{d T}{d t}=a_{1} \cdot\left(T_{0}-T\right)+a_{2} \cdot k_{1} \cdot c_{A}+a_{3} \cdot q_{c} \cdot\left(1-e^{\frac{a_{4}}{q_{c}}}\right) \cdot\left(T_{0}-T\right) \\
\frac{d c_{A}}{d t}=a_{1} \cdot\left(c_{A 0}-c_{A}\right)-k_{1} \cdot c_{A}
\end{gathered}
$$


where $a_{1-4}$ are constants computed as

$$
a_{1}=\frac{q}{V} ; a_{2}=\frac{-\Delta H}{\rho \cdot c_{p}} ; a_{3}=\frac{\rho_{c} \cdot c_{p c}}{\rho \cdot c_{p} \cdot V} ; a_{4}=\frac{-h_{a}}{\rho_{c} \cdot c_{p c}} \text { (2) }
$$

variable $t$ in previous equations denotes time, $T$ is used for temperature of the reactant, $V$ is volume of the reactor, $c_{A}$ represents concentration of the product, $q$ and $q_{c}$ are volumetric flow rates of the reactant and cooling respectively. Indexes $(\cdot)_{0}$ denote inlet values of the variables and $(\cdot)_{c}$ is used for variables related to the cooling. The fixed values of the system are shown in Table 1 (Gao et al. 2002).

The nonlinearity of the model can be found in relation for the reaction rate, $k_{1}$, which is computed from Arrhenius law:

$$
k_{1}=k_{0} \cdot \mathrm{e}^{\frac{-E}{R \cdot T}}
$$

where $k_{0}$ is reaction rate constant, $E$ denotes an activation energy and $R$ is a gas constant.

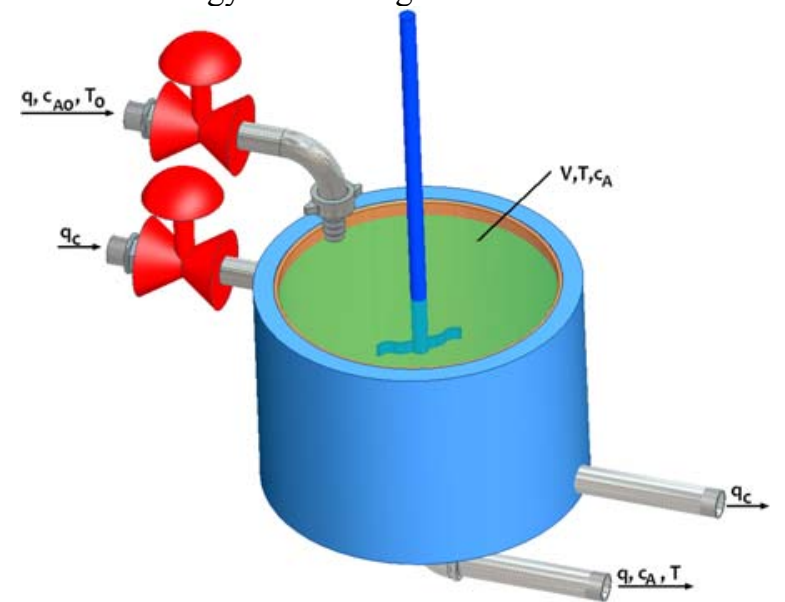

Figure 1: Continuous Stirred Tank Reactor

\begin{tabular}{|c|c|}
\hline $\begin{array}{l}\text { Reactant's flow rate } \\
\text { Reactor's volume } \\
\text { Reaction rate constant } \\
\text { Activation energy to R } \\
\text { Reactant's feed temperature } \\
\text { Reaction heat } \\
\text { Specific heat of the reactant } \\
\text { Specific heat of the cooling } \\
\text { Density of the reactant } \\
\text { Density of the cooling } \\
\text { Feed concentration } \\
\text { Heat transfer coefficient }\end{array}$ & $\begin{array}{c}q=100 \mathrm{l}_{\mathrm{min}}^{-1} \\
V=100 \mathrm{l} \\
k_{0}=7.2 \cdot 10^{10} \mathrm{~min}^{-1} \\
E / R=1 \cdot 10^{4} \mathrm{~K} \\
T_{0}=350 \mathrm{~K} \\
\Delta H=-2 \cdot 10^{5} \mathrm{cal} . \mathrm{mol}^{-1} \\
c_{p}=1 \mathrm{cal} . \mathrm{g}^{-1} \cdot \mathrm{K}^{-1} \\
c_{p c}=1 \mathrm{cal} . \mathrm{g}^{-1} \cdot \mathrm{K}^{-1} \\
\rho=1 \cdot 10^{3} \mathrm{~g} \cdot \mathrm{l}^{-1} \\
\rho_{c}=1 \cdot 10^{3} \mathrm{~g} \cdot \mathrm{l}^{-1} \\
c_{A 0}=1 \mathrm{~mol} . \mathrm{l}^{-1} \\
h_{a}=7 \cdot 10^{5} \mathrm{cal} . \mathrm{min}^{-1} \cdot \mathrm{K}^{-1}\end{array}$ \\
\hline
\end{tabular}

Table 1: Fixed parameters of the reactor

\section{STEADY-STATE AND DYNAMIC ANALYSES}

The steady-state and dynamic analyses presented in (Vojtesek and Dostal 2008) have shown that the system has nonlinear behaviour and we cannot choose the exact optimal working point. The resulting working point is then combination of the possibly lowest values of the volumetric flow rates $q$ and $q_{c}$ :

$$
q_{c}=80 l . \mathrm{min}^{-1} \quad q=100 \mathrm{l} \cdot \mathrm{min}^{-1}
$$

The steady-state values of state variables $T$ and are $c_{A}$ for this working point

$$
T^{s}=354.26 K \quad c_{A}^{s}=0.9620 \mathrm{~mol}^{-l^{-1}}
$$

The dynamic analysis for both outputs presented in the same paper (Vojtesek and Dostal 2008) shows that the output temperature could be described by the first or the second order transfer function and the second order transfer function could be used as a description of the output concentration $c_{A}$

\section{ADAPTIVE CONTROL}

The controlled variable was product's temperature $T$ related to its steady-state value and the following simulation studies were done for both possible input volumetric flow rates of the reactant $q$ and cooling $q_{c}$, i.e.

$$
\begin{aligned}
& u_{1}(t)=\frac{q_{c}(t)-q_{c}^{s}}{q_{c}^{s}} \cdot 100[\%] \\
& u_{2}(t)=\frac{q(t)-q^{s}}{q^{s}} \cdot 100[\%]
\end{aligned}
$$

\section{External Linear Model(ELM)}

Although the original system has nonlinear behaviour, the External Linear Model (ELM) is used as a reprentation of the controlled system.

As it is written above, the controlled output could be described by the second order transfer function with relative order one:

$$
G(s)=\frac{Y(s)}{U(s)}=\frac{b(s)}{a(s)}=\frac{b_{1} s+b_{0}}{s^{2}+a_{1} s+a_{0}}
$$

This transfer function fulfils the condition of properness $\operatorname{deg} b \leq \operatorname{deg} a$.

ELM described as (7) belongs to the class of continuous-time (CT) models. The identification of such processes is not very easy. The solution to this problem can be found in the use of so called $\delta$-model which belongs to the class of discrete models but its parameters are close to the continuous ones for very small sampling period (Stericker and Sinha 1993).

The $\delta$-model introduces a new complex variable $\gamma$ computed as (Mukhopadhyay et al. 1992)

$$
\gamma=\frac{z-1}{\beta \cdot T_{v} \cdot z+(1-\beta) \cdot T_{v}}
$$

And it is obvious, that we can obtain infinitely many models for optional parameter $\beta$ from the interval $0 \leq \beta \leq 1$ and a sampling period $T_{v}$, however a forward $\delta$-model was used in this work which has $\gamma$ operator computed via

$$
\beta=0 \Rightarrow \gamma=\frac{z-1}{T_{v}}
$$


The differential equation for ELM in the form of (7) is

$$
\begin{aligned}
y_{\delta}(k)= & -a_{1} y_{\delta}(k-1)-a_{0} y_{\delta}(k-2)+ \\
& +b_{1} u_{\delta}(k-1)+b_{0} u_{\delta}(k-2)
\end{aligned}
$$

where $y_{\delta}$ is the recomputed output to the $\delta$-model:

$$
\begin{aligned}
& y_{\delta}(k)=\frac{y(k)-2 y(k-1)+y(k-2)}{T_{v}^{2}} \\
& y_{\delta}(k-1)=\frac{y(k-1)-y(k-2)}{T_{v}} \\
& y_{\delta}(k-2)=y(k-2) \\
& u_{\delta}(k-1)=\frac{u(k-1)-u(k-2)}{T_{v}} \\
& u_{\delta}(k-2)=u(k-2)
\end{aligned}
$$

and $T_{v}$ is a sampling period, the data vector is then

$$
\begin{array}{r}
\phi_{\delta}^{T}(k-1)=\left[-y_{\delta}(k-1),-y_{\delta}(k-2), \ldots\right. \\
\left.\ldots, u_{\delta}(k-1), u_{\delta}(k-2)\right]
\end{array}
$$

The vector of estimated parameters

$$
\hat{\boldsymbol{\theta}}_{\delta}^{T}(k)=\left[a_{1}^{\delta}, a_{0}^{\delta}, b_{1}^{\delta}, b_{0}^{\delta}\right]
$$

can be computed from the ARX (Auto-Regressive eXtrogenous) model

$$
y_{\delta}(k)=\theta_{\delta}^{T}(k) \cdot \varphi_{\delta}(k-1)
$$

by some of the recursive least squares methods.

The Recursive Least-Squares (RLS) method used for parameter estimation of the vector $\hat{\theta}_{\delta}^{T}$ is well-known and widely used identification method (Fikar and Mikles, 1999). The ordinary RLS method is described by the set of equations:

$$
\begin{gathered}
\varepsilon(k)=y(k)-\boldsymbol{\varphi}^{T}(k) \cdot \hat{\boldsymbol{\theta}}(k-1) \\
\gamma(k)=\left[1+\boldsymbol{\varphi}^{T}(k) \cdot \mathbf{P}(k-1) \cdot \boldsymbol{\varphi}(k)\right]^{-1} \\
\boldsymbol{L}(k)=\gamma(k) \cdot \mathbf{P}(k-1) \cdot \boldsymbol{\varphi}(k) \\
\mathbf{P}(k)=\mathbf{P}(k-1)-\gamma(k) \cdot \mathbf{P}(k-1) \cdot \boldsymbol{\varphi}(k) \\
\boldsymbol{\varphi}^{T}(k) \cdot \mathbf{P}(k-1) \\
\hat{\boldsymbol{\theta}}(k)=\hat{\boldsymbol{\theta}}(k-1)+\boldsymbol{L}(k) \varepsilon(k)
\end{gathered}
$$

Where $\varepsilon$ denotes a prediction error and $\mathbf{P}$ is a covariance matrix. This ordinary RLS method could be modified with some kind of forgetting, exponential or directional but simulation experiments have shown, that there is no need to use these modifications in this case .

\section{Configuration of the Controller}

Two control system configurations with one degree-offreedom (1DOF) and two degrees-of-freedom (2DOF) were used here. The first, 1DOF, configuration has controller in the feedback part - see Figure 2.

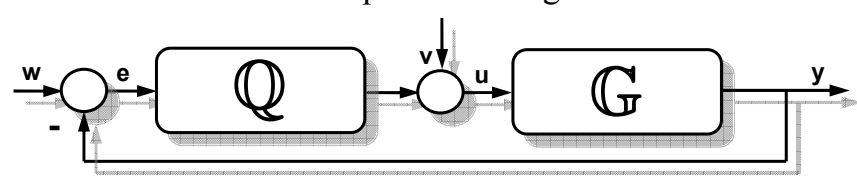

Figure 2: 1DOF control configuration
The configuration with two degrees-of-freedom (2DOF) displayed in Figure 3 has controller divided into feedback part (Q) and feedforward part (R).

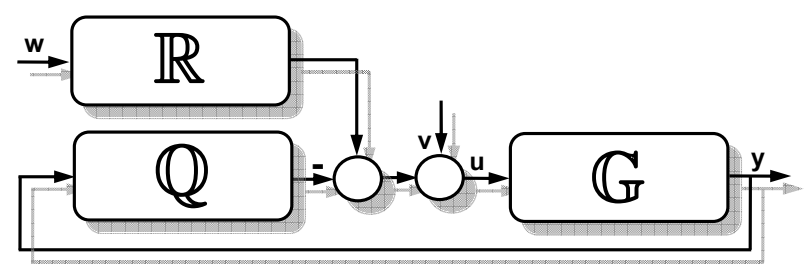

Figure 3: 2DOF control configuration

$G$ in both configurations denotes transfer function (7) of controlled plant, $w$ is the reference signal (wanted value), $v$ is disturbance, $e$ is used for control error, $u$ is control variable and $y$ is a controlled output.

The feedback and feedforward parts of the controller are designed with the use of polynomial synthesis:

$$
Q(s)=\frac{q(s)}{s \cdot p(s)} ; R(s)=\frac{r(s)}{s \cdot p(s)}
$$

where parameters of the polynomials $p(s), q(s)$ and $r(s)$ are computed by the Method of uncertain coefficients which compares coefficients of individual $s$-powers from Diophantine equations (Kucera, 1993):

$$
\begin{gathered}
a(s) \cdot s \cdot p(s)+b(s) \cdot q(s)=d(s) \\
t(s) \cdot s+b(s) \cdot r(s)=d(s)
\end{gathered}
$$

The resulted, so called "hybrid", controller works in the continuous time but parameters of the polynomials $a(s)$ and $b(s)$ are identified recursively in the sampling period $T_{v}$. It is clear, that the $1 \mathrm{DOF}$ control configuration counts only with the first equation in (17) while 2DOF configuration needs both Diophantine equations. Polynomial $t(s)$ in the second Diophantine equation is an additive stable polynomial with random coefficients, because these coefficients are not used for computing of coefficients of the polynomial $r(s)$ in $2 \mathrm{DOF}$ configuration. All these equations are valid for step changes of the reference and disturbance signals.

The feedback controller $Q(s)$ ensures stability, load disturbance attenuation for both configurations and asymptotic tracking for $1 \mathrm{DOF}$ configuration. On the other hand, feedforward part $R(s)$ ensures asymptotic tracking in 2DOF configuration.

The polynomial $d(s)$ on the right side of (17) is an optional stable polynomial. Roots of this polynomial are called poles of the closed-loop and their position affects quality of the control.

This polynomial could be designed for example with the use of Pole-placement method. The degree of the polynomial $d(s)$ is in this case

$$
\operatorname{deg} d(s)=\operatorname{deg} a(s)+\operatorname{deg} \tilde{p}(s)+1
$$

A choice of the roots needs some a priory information about the system's behaviour. It is good to connect poles with the parameters of the system via spectral factorization. The polynomial $d(s)$ then for our ELM (7) can be rewritten for aperiodical processes to the form 


$$
d(s)=n(s) \cdot(s+\alpha)^{\operatorname{deg} d-\operatorname{deg} n}
$$

where $\alpha>0$ is an optional coefficient reflecting closedloop poles and stable polynomial $n(s)$ is obtained from the spectral factorization of the polynomial $a(s)$

$$
n^{*}(s) \cdot n(s)=a^{*}(s) \cdot a(s)
$$

The degrees of the polynomials $p(s), q(s)$ and $r(s)$ are for this second order ELM deg $q(s)=2, \operatorname{deg} q(s)=1$ and $\operatorname{deg} r(s)=1$, which means that

$$
\begin{aligned}
& \tilde{Q}(s)=\frac{q(s)}{s \cdot \tilde{p}(s)}=\frac{q_{2} s^{2}+q_{1} s+q_{0}}{s \cdot\left(s+p_{0}\right)} \\
& \tilde{R}(s)=\frac{r(s)}{s \cdot \tilde{p}(s)}=\frac{r_{0}}{s \cdot\left(s+p_{0}\right)}
\end{aligned}
$$

and the polynomial $d(s)$ is from (19) of the fourth degree and it could be chosen as

$$
d(s)=n(s) \cdot(s+\alpha)^{2}
$$

Parameters of the polynomial $n(s)$ which are computed from the spectral factorization are defined as:

$$
n_{0}=\sqrt{a_{0}^{2}}, n_{1}=\sqrt{a_{1}^{2}+2 n_{0}-2 a_{0}}
$$

\section{SIMULATION RESULTS OF CONTROL}

Several simulation studies were done on this system. All of them were done in the mathematical software Matlab, version 6.5.1 and the common values for all simulations were: the sampling period was $T_{v}=0.3 \mathrm{~min}$, the simulation time $1000 \mathrm{~min}$ and 5 different step changes were done during this time.

The input variables $u_{1}(t)$ and $u_{2}(t)$ were limited to the bounds $<-90 \% ;+90 \%>$. The initial vector of parameters used for identification was $\hat{\boldsymbol{\theta}}^{T}=[0.1,0.1,0.1,0.1]$ and the initial covariance matrix was $\boldsymbol{P}_{i i}=1 \cdot 10^{7}$ for $i=1, . ., 4$.

The quality of control was evaluated by the quality criteria $S_{u}$ and $S_{y}$ computed for a whole time interval as

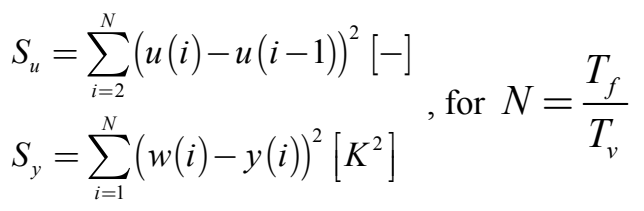

The first simulation study compares controllers with $1 \mathrm{DOF}$ and $2 \mathrm{DOF}$ control configutations for change of the volumetric flow rate of coolant, $q_{c}$, as an input variable. It is good to have opurtunity to affect the control output somehow. The tuning parameter in our case is position of the root $\alpha$. And the effect of the different $\alpha=0.02,0.05$ and 0.09 is studied here. Results are shown in following figures.

Courses of the output variable $y(t)$ and the input variable $u_{1}(t)$ presented in Figure 4 and Figure 5 show, that the decreasing value of the tuning parameter $\alpha$ results in slower output response and output does not reach reference signal $w(t)$ in $200 \mathrm{~min}$ which is used for each step change. On the other hand, course of the input variable is smoother for lower value of $\alpha$.

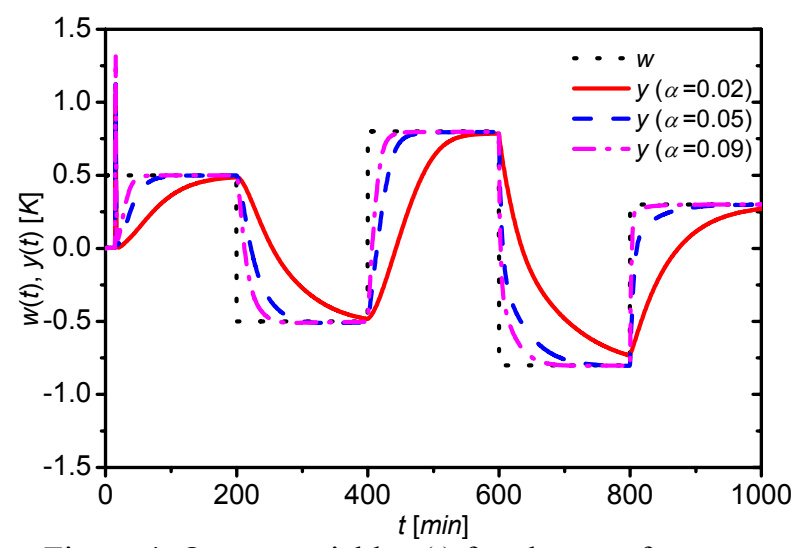

Figure 4: Output variable $y(t)$ for change of $q_{c}$ as an input, $1 \mathrm{DOF}$ and various values of tuning parameter $\alpha$

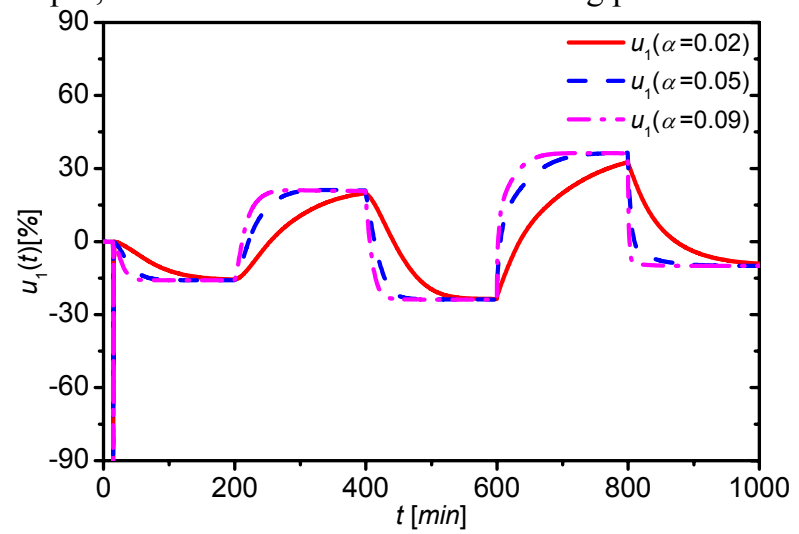

Figure 5: Input variable $u_{1}(t)$ for $1 \mathrm{DOF}$ and various values of tuning parameter $\alpha$

The only control problem can be found at the very beginning which is caused by the identification, which does not have enough information and need some time to estimate parameters of the system's transfer function. However, output response in the following simulation time is smooth and without any problems - see course of the identified parameters in Figure 6 and Figure 7.

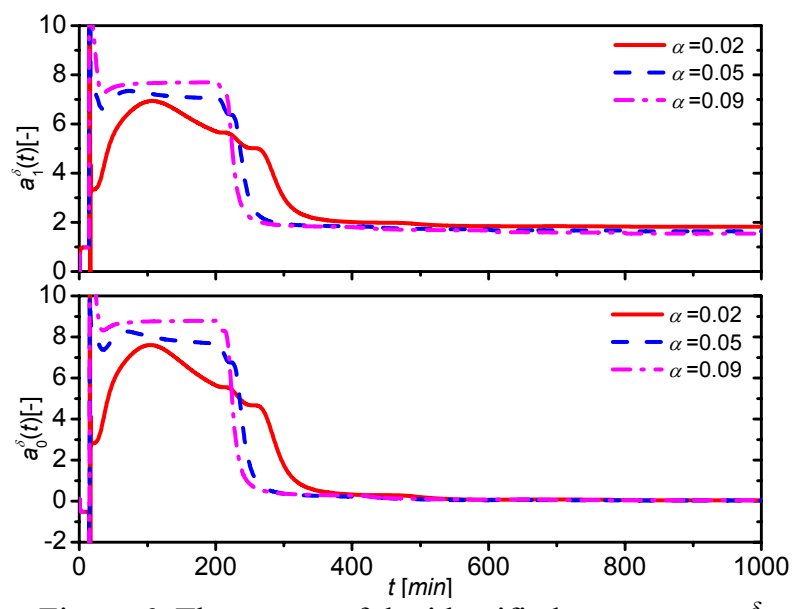

Figure 6: The course of the identified parameters $a_{1}^{\delta}$ and $a_{0}^{\delta}$, 1DOF configuration and various values of tuning parameter $\alpha$ 


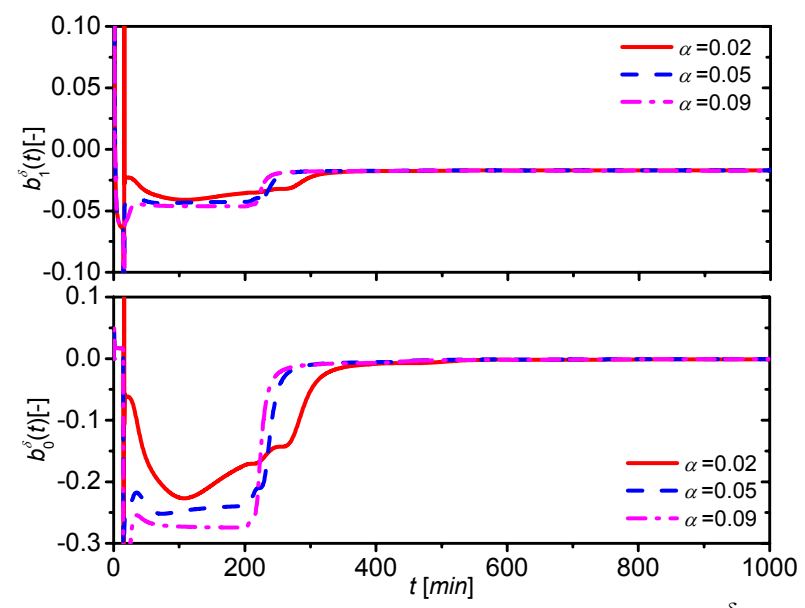

Figure 7: The course of the identified parameters $b^{\delta}$ and $b_{0}^{\delta}, 1 \mathrm{DOF}$ configuration and various values of tuning parameter $\alpha$

The second simulation study observes effects of 2DOF control configuration on the controller output. The course of the output temperature represented by the output $y(t)$ shows a bit worse results especially at the beginning of the control caused by the identification againg. The control results after approximately $100 \mathrm{~min}$ are again quite good which is displayed in Figure 8 and Figure 9.

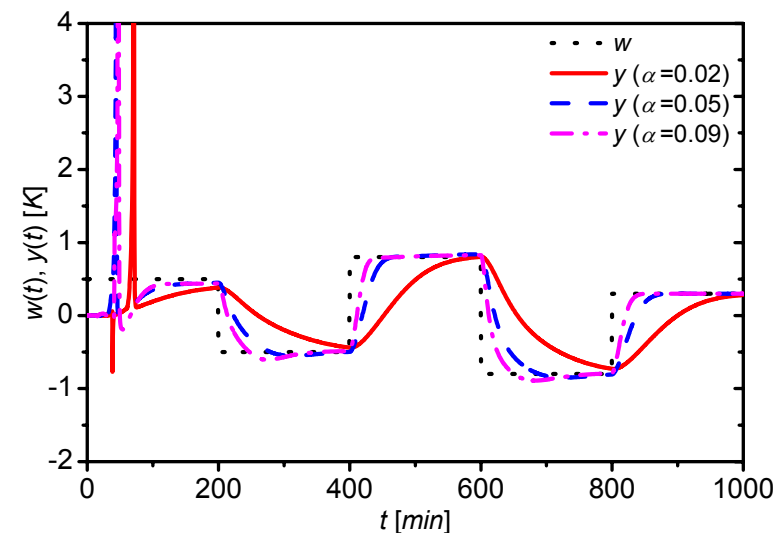

Figure 8: Output variable $y(t)$ for change of $q_{c}$ as an input, 2DOF and various values of tuning parameter $\alpha$

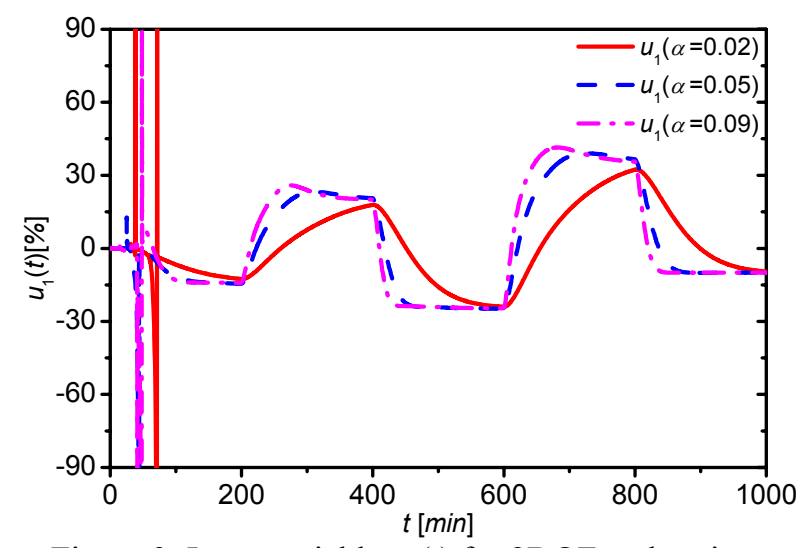

Figure 9: Input variable $u_{1}(t)$ for $2 \mathrm{DOF}$ and various values of tuning parameter $\alpha$
Both, 1DOF and 2DOF, control configurations are compared in Figure 10 and Figure 11 for the tuning parameter $\alpha=0.09$. The main difference can be found in first $100 \mathrm{~min}$ which was already mentioned and the 2DOF control configuration has small overshoots for step changes from the bigger value to lower.

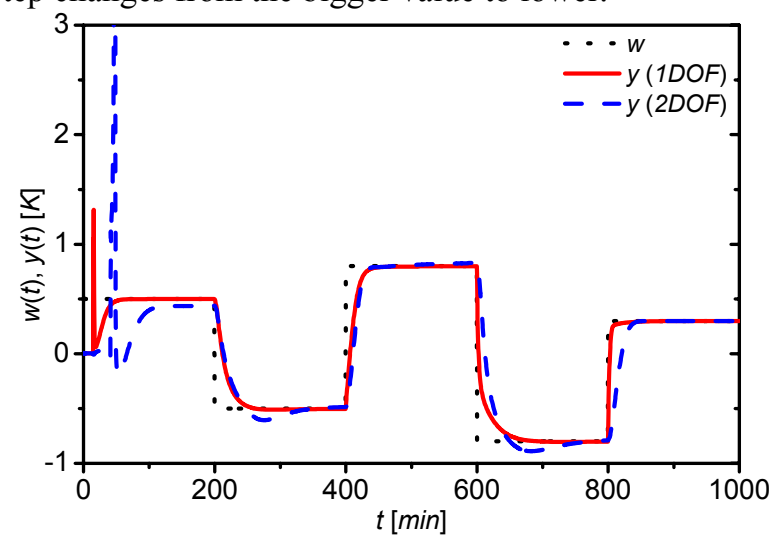

Figure 10: Comparison of 1DOF and 2DOF configuration, course of the output variable $y(t)$, change

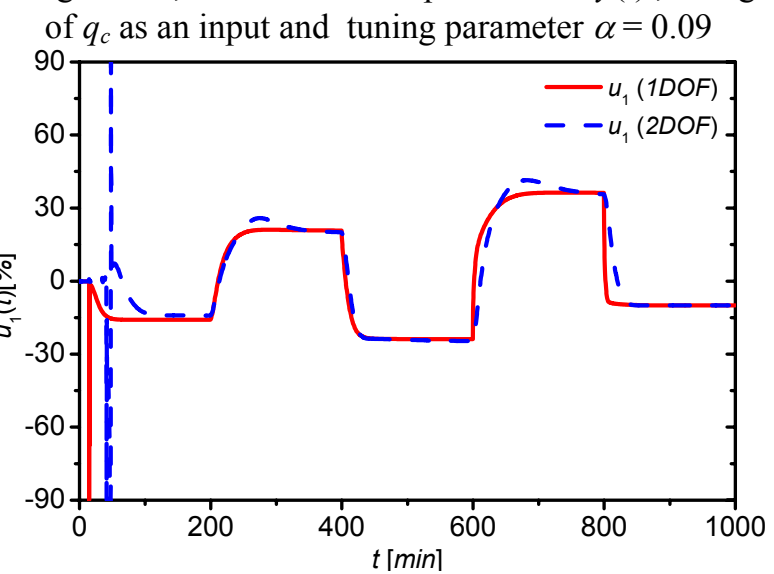

Figure 11: Comparison of 1DOF and 2DOF configuration, course of the input variable $u_{1}(t)$ and tuning parameter $\alpha=0.09$

The third study, results of which are displayed in Figure 12, Figure 13 and Figure 14, have been done for the different input $u_{2}(t)$ which is change of the input volumetric flow rate of the reactant $q$.

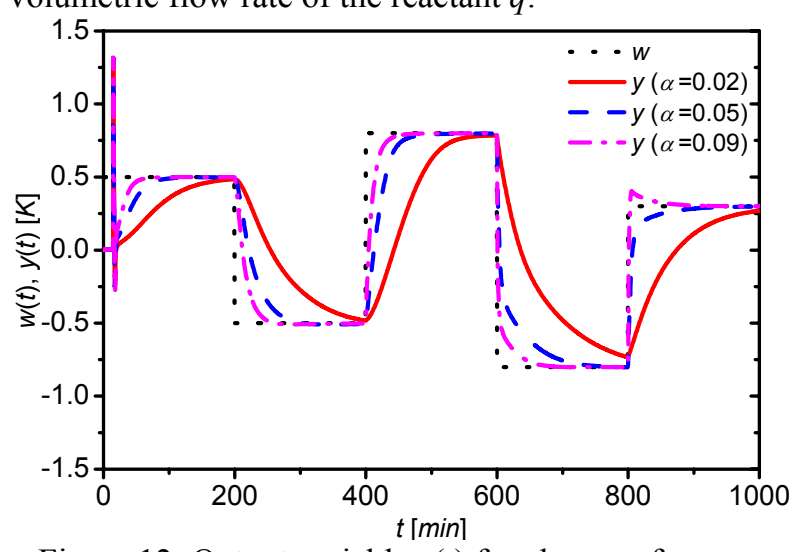

Figure 12: Output variable $y(t)$ for change of $q$ as an input, 1DOF and various values of tuning parameter $\alpha$ 
The results clearly implies that proposed control strategy could be used for this input too with similar results. The $2 \mathrm{DOF}$ configuration provides worse results especially at the beginning of the control and after the second step change too - see Figure 13 and Figure 14.

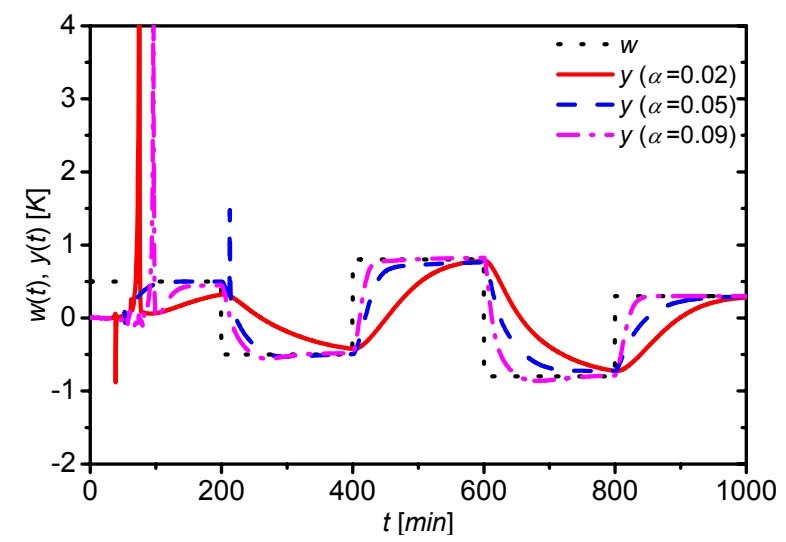

Figure 13: Output variable $y(t)$ for change of $q$ as an input, 2DOF and various values of tuning parameter $\alpha$

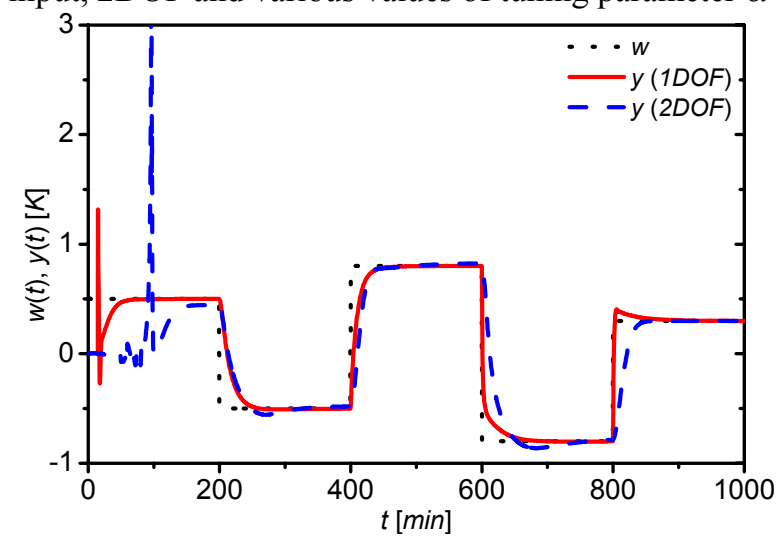

Figure 14: Comparison of 1DOF and 2DOF configuration, course of the output variable $y(t)$, change of $q$ as an input and tuning parameter $\alpha=0.09$

The values of control quality criterions $\mathrm{Su}$ and $\mathrm{Sy}$ for both input variables $u_{1}$ for $q_{c}$ and $u_{2}$ for $q, 1 \mathrm{DOF}$ and 2DOF control configurations are shown in Table 2 and Figure 15 and Figure 16.

Table 2: Values of control quality criteria $S_{u}$ and $S_{y}$

\begin{tabular}{|r|r|r|r|r|}
\cline { 2 - 5 } \multicolumn{1}{c|}{} & \multicolumn{1}{c|}{ 1DOF } & \multicolumn{2}{c|}{ 2DOF } \\
\cline { 2 - 5 } \multicolumn{1}{c|}{} & \multicolumn{1}{c|}{$S_{u}[-]$} & $S_{v}\left[K^{2}\right]$ & \multicolumn{1}{c|}{$S_{u}[-]$} & $S_{v}\left[K^{2}\right]$ \\
\hline \multicolumn{4}{|c|}{ Results for $u_{1}(t)-$ volumetric flow rate $q_{c}$} \\
\hline$\alpha=0.02$ & 20.85 & 690.79 & 15.59 & 949.30 \\
$\alpha=0.05$ & 144.31 & 198.21 & 49.26 & 377.28 \\
$\alpha=0.09$ & 498.59 & 94.39 & 84.72 & 216.81 \\
\hline \multicolumn{6}{|c|}{ Results for $u_{2}(t)-$ volumetric flow rate q } \\
\hline$\alpha=0.02$ & 15.04 & 695.03 & 10.89 & 934.83 \\
$\alpha=0.05$ & 146.63 & 174.13 & 14.442 & 409.28 \\
$\alpha=0.09$ & 909.11 & 71.36 & 60.60 & 213.43 \\
\hline
\end{tabular}

The values of criterions $S_{u}$ and $S_{y}$ from (24) are computed not from the very beginning, but after the first step change, i.e. from time $200 \mathrm{~min}$ due to inaccurate identification at the very beginning. This table and graphs verify previous statements, that increasing value of a results in quicker output response - see decreasing value of $S_{y}$ but value of $S_{u}$ representing the change of input variable increase.

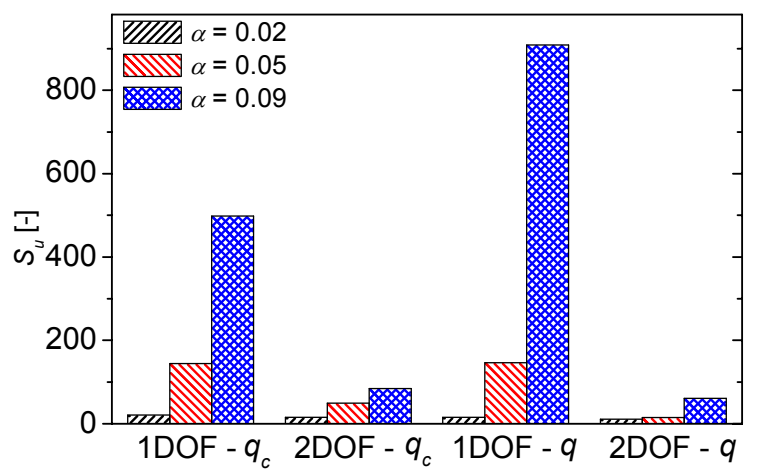

Figure 15: Values of control quality criterion $S_{u}$ for all simulation studies

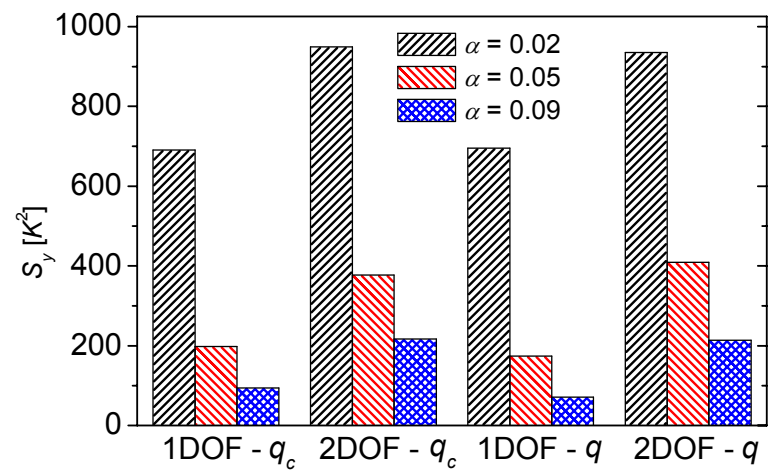

Figure 16: Values of control quality criterion $S_{y}$ for all simulation studies

The proposed controller fulfills basic control requierements and one of it is disturbance attenuation. The last study provides proof to this statement. Simulation took $600 \mathrm{~min}$, one step change of the reference signal $w(t)$ was done during this time and three step disturbances are inceted to the system - two on the input and one on the output:

- $v_{1}(t)=+10 \%$ step change of the input concentration $c_{A 0}$ for time $t \in\langle 150 ; 600\rangle \mathrm{min}$

- $v_{2}(t)=-1.5 \mathrm{~K}$ step change of the input reactant's temperature $T_{0}$ for time $t \in\langle 300 ; 600\rangle \mathrm{min}$

- $v_{2}(t)=+0.4 K$ step change of the output product's temperature $T$ for time $t \in\langle 450 ; 600\rangle \mathrm{min}$

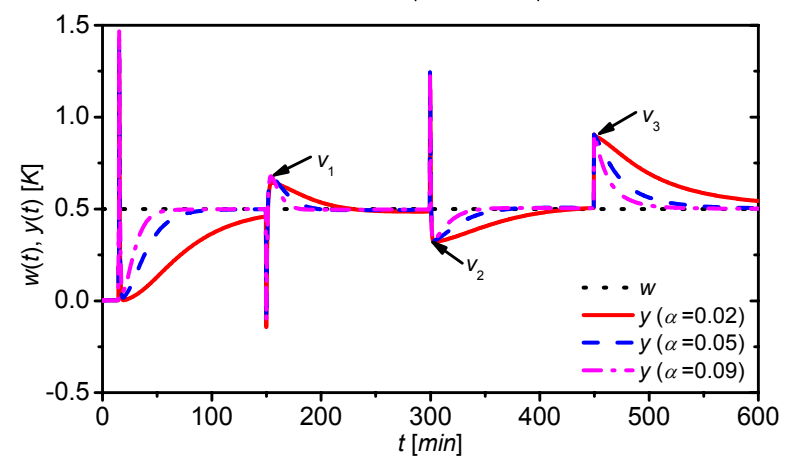

Figure 17: Course of the output variable $y(t)$ for disturbances and various values of tuning parameter $\alpha$ 
As you can see in Figure 17 and Figure 18. adaptive controller has no problem with the disturbance attenuation. Note that all three disturbances affects together in the last interval from 450 to $600 \mathrm{~min}$ and controller dealt with it quite good.

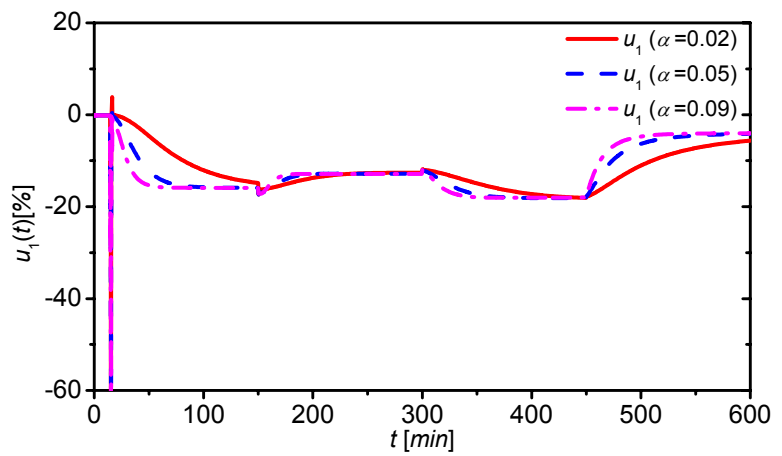

Figure 18: Course of the input variable $u_{1}(t)$ for disturbances and various values of tuning parameter $\alpha$

\section{CONCLUSION}

This paper presents simulation studies on the CSTR focused especially on the adaptive control. Mathematical model of system is described by the set two nonlinear ordinary differential equations which are solved numerically by the Runge-Kutta's standard method. Steady-state and dynamic analyses result in the working point and the choice of the second order transfer function with relative order one as a ELM of the originally nonlinear process. The control results for both $1 \mathrm{DOF}$ and 2DOF control configuration presents good course of the product's temperature as an output variable. The course of this output can be affected by the choice of the root $\alpha$ and we can say, that increasing value of this root results in quicker response. The only disadvantage can be found in inadequate behaviour at the begging of the control which is cased by the absence of the initial information about the system. The system could have two input variables but simulations have shown that the change of the volumetric flow rate of the cooling, $q_{c}$, have a bit better control results than the change of the volumetric flow rate of the reactant, $q$.

The proposed control strategies satisfies basic control requirements which was proofed in the last study where three disturbances were injected into the system but the results are again quite good.

\section{REFERENCES}

Åström, K.J. and B. Wittenmark 1989. Adaptive Control. Addison Wesley. Reading. MA.

Bobal, V.; J. Böhm; J. Fessl; J. Machacek 2005. Digital Selftuning Controllers: Algorithms. Implementation and Applications. Advanced Textbooks in Control and Signal Processing. Springer-Verlag London Limited

Fikar, M.; J. Mikles 1999. System Identification. STU Bratislava

Gao, R.; A. O'dywer; E. Coyle 2002. A Non-linear PID Controller for CSTR Using Local Model Networks. Proc. of $4^{\text {th }}$ World Congress on Intelligent Control and Automation. Shanghai. P. R. China. 3278-3282

Ingham, J.; I. J. Dunn; E. Heinzle; J. E. Přenosil 2000. Chemical Engineering Dynamics. An Introduction to Modeling and Computer Simulation. Second. Completely Revised Edition. VCH Verlagsgesellshaft. Weinheim.

Kucera, V. 1993. Diophantine equations in control - A survey. Automatica. 29. 1361-1375

Middleton, R.H.; G. C. Goodwin 2004 Digital Control and Estimation - A Unified Approach. Prentice Hall. Englewood Cliffs.

Mukhopadhyay, S.; A. G. Patra; G. P. Rao 1992. New class of discrete-time models for continuos-time systeme. International Journal of Control. vol.55. 1161-1187

Stericker, D.L; N. K. Sinha 1993. Identification of continuous-time systems from samples of input-output data using the $\delta$-operator. Control-Theory and Advanced Technology. vol. 9. 113-125

Vojtesek, J; P. Dostal 2008. Simulation analyses of continuous stirred tank reactor. In: Proceedings of the 22nd European Conference on Modelling and Simulation. Nicosia, Cyprus. p. 506-511.

\section{ACKNOWLEDGMENT}

This work was supported by the Ministry of Education of the Czech Rep. under grants No. MSM 7088352101 and MSM 7088352102.

\section{AUTHOR BIOGRAPHIES}

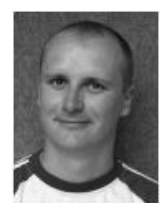

JIRI VOJTESEK was born in Zlin. Czech Republic and studied at the Tomas Bata University in Zlin. where he got his master degree in chemical and process engineering in 2002. He has finished his Ph.D. focused on Modern control methods for chemical reactors in 2007. His contact is vojtesek@fai.utb.cz.

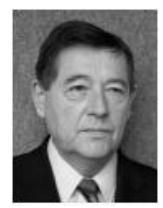

PETR DOSTAL studied at the Technical University of Pardubice. He obtained his PhD. degree in Technical Cybernetics in 1979 and he became professor in Process Control in 2000. His research interest are modeling and simulation of continuous-time chemical processes. polynomial methods. optimal. adaptive and robust control. You can contact him on email address dostalp@fai.utb.cz.

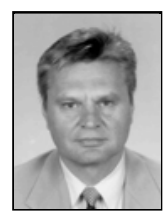

ROMAN PROKOP was born in Hodonín, Czech Republic in 1952. He graduated in Cybernetics from the Czech Technical University in Prague in 1976. He received post graduate diploma in 1983 from the Slovak Technical University. Since 1995 he has been at Tomas Bata University in Zlín, where he presently holds the position of full professor of the Department of Automation and Control Engineering and a vice-dean of the Faculty of Applied Informatics. His research activities include algebraic methods in control theory, robust and adaptive control, autotuning and optimization techniques. His e-mail address is: prokopefai.utb.cz. 